\title{
Laser Speckle Imaging of Cerebral Blood Flow
}

\author{
Pavel Zakharov, Frank Scheffold, and Bruno Weber
}

\begin{abstract}
Optical imaging of cerebral hemodynamics has been used extensively to investigate the complex interplay between neurons and the blood-borne supply of oxygen and energy substrates. Among several techniques, the so-called laser speckle imaging (LSI) has found widespread applications for studying (patho-)physiological aspects of neurovascular coupling. LSI is a rather simple and cost-effective method to acquire two-dimensional maps of cerebral blood flow from the cortex of experimental animals. In this chapter, we introduce the physical underpinnings as well as the most important technical and practical aspects of its implementation.
\end{abstract}

Key words Optical imaging, Cerebral blood flow, Laser speckle, Hemodynamics, Laser, In vivo, Somatosensory system, Barrel cortex

1 Introduction

Early on, high-resolution functional neuroimaging with optical tools was tightly linked to cerebral blood flow (CBF). Indeed, pioneering work of Chance et al. [1] and Jöbsis [2] demonstrated that the intrinsic properties of the chromophores and their relationship to hemodynamics could be applied for monitoring neuronal activity. This work paved the way for the first high-resolution optical mapping of hemodynamic signals by the group of Amiram Grinvald using charge-coupled device (CCD) technology [3, 4]. Since then, optical imaging of intrinsic signals (OIS) has been extensively applied for studying cortical processing. Apart from using OIS as a surrogate for neuronal activity and thereby for mapping cortical processing, optical imaging became an important tool for investigating neurovascular coupling. This research field has grown considerably during the last 2 decades, as the processes governing the regulation of $\mathrm{CBF}$ are still far from being understood (for a recent review, see Attwell et al. [5]). Many of the widely used noninvasive imaging tools to study the human brain in actionsuch as blood oxygenation level-dependent (BOLD) functional 
magnetic resonance imaging-rely on hemodynamic signals. Furthermore, a number of the most relevant neurological diseases (such as stroke or Alzheimer's disease) show abnormalities in vascular structure and function [6]. Although OIS is based on the hemodynamic response that follows an increase in neuronal activity (see also Chapter 14 in this volume), it is important to note that blood oxygenation and $\mathrm{CBF}$ are by no means identical. Interestingly, changes in blood oxygenation and $\mathrm{CBF}$ can be uncoupled in certain (patho-)physiological conditions. For example, Lindauer et al. [7] could show that increased baseline blood oxygenation caused by hyperbaric hyperoxia can completely abolish the deoxy- $\mathrm{Hb}$ response, while the $\mathrm{CBF}$ response remains unchanged. For many questions related to neurovascular coupling and pathophysiological states, direct measurements of CBF are thus mandatory. This is the main reason for today's usage of complementary optical methods that directly monitor CBF. In this field laser speckle imaging (LSI) has found widespread application, as it allows imaging of local changes in $\mathrm{CBF}$ with rather high spatial and temporal resolution [8-11].

LSI is an implementation of the family of methods based on quasi-elastic light scattering (QELS). The method employs the statistical analysis of temporal fluctuations of light scattered by microscale dynamic scattering heterogeneities in order to extract quantitative information about their dynamics. The motion of scattering objects, such as red blood cells (RBCs), results in Doppler shifts, which are minuscule as compared to the frequency of light. Thus the scattering is coined "quasi-elastic" meaning that the light energy is essentially conserved throughout the whole process.

The frequency-shifted light is further optically mixed with the light scattered by the static tissues. If within the volume under observation the scatterers move in random directions with fluctuating velocity the Doppler shifts add up and a Doppler broadening is observed rather than a shift. Such random motion can be due to the intrinsic Brownian motion but can also be mimicked by the complex blood flow pattern in vascularized tissue. For the case of the cortical microvascular system which lacks defined flow directions the light reaching the photodetector produces the noise-like fluctuations of the photocurrent with the spectral properties defined by the scattering geometry, number of scattering particles (typically RBCs), and their velocity. It can be shown [12] that the first moment of the power spectrum is proportional to product of scatterer concentration and average velocity, while the zero-th moment is proportional to the concentration. The variations of photocurrent are typically processed with the spectrum analyzer, which provides the estimation of the spectral moments. The technique is usually referred to as Laser Doppler Velocimetry (LDV) or flowmetry (LDF). 

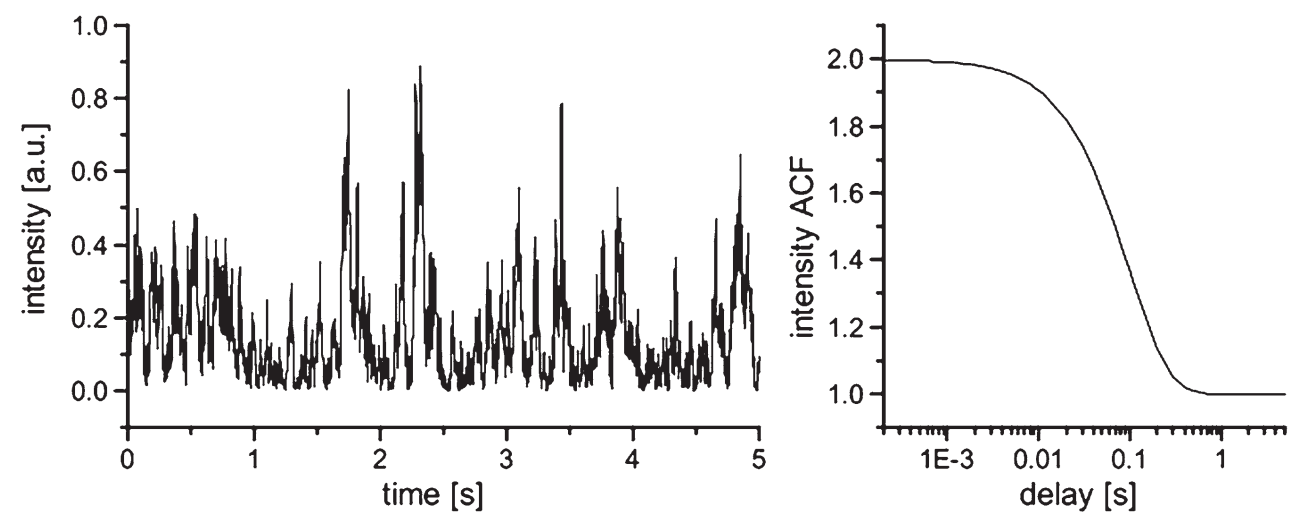

Fig. 1 Illustration of the intensity fluctuations caused by the movement of scattering particles (left) and corresponding intensity correlation function (right). From [15].

For this case the narrow line-width of incident laser light is slightly broadened due to motion, which can be directly detected by (Doppler-) spectroscopy but can also be regarded as time fluctuating random diffraction pattern. The latter is known as speckle and it is fluctuating due to changes in position of the scatterers within the scattering volume. Such fluctuations can be analyzed in the time domain with the help of the intensity correlation function, which is related to the frequency power spectrum via the Fourier transform (Wiener-Khinchin theorem) [13]. A hardware or software correlator can be used for the analysis of these fluctuations. The resulting temporal fluctuations of light intensities in the millisecond range are easily analyzed while the corresponding spectral broadening of the order of $\Delta f=1 / \tau \sim 1 \mathrm{kHz}$ is often more difficult to measure. The formalism outlined in the next section can be further applied to single or multiple scattering alike. The former is often called "dynamic light scattering" (DLS) while the latter is know under the name of "diffusing wave spectroscopy" (DWS) [14].

In general, in QELS the temporal fluctuations of scattered light intensity $I(t)$ are measured (see Fig. 1) and the intensity correlation function (ICF) is calculated:

$$
g_{2}(\tau)=\frac{\langle I(t) I(t+\tau)\rangle_{t}}{\langle I(t)\rangle_{t}^{2}}
$$

where $\langle\cdots\rangle_{t}$ denotes time averaging. The physical meaning is attributed to the field $E(t)$ correlation function $g_{1}(\tau)$ :

$$
g_{1}(\tau)=\frac{\left\langle E(t) E^{*}(t+\tau)\right\rangle_{t}}{\langle|E(t)|\rangle_{t}^{2}},
$$

which is related to the measurable ICF via the Siegert relation [16]:

$$
\mathfrak{g}_{2}(\tau)=1+\beta\left|\mathfrak{g}_{1}(\tau)\right|^{2},
$$


where $\beta$ is usually referred to as coherence factor and depends on the detection conditions. In an ideal case when single speckle mode is detected it is approaching the value of 1 . In the case of complete loss of coherence $\beta=0$.

While the approaches to data analysis of Doppler and DLS/DWS methods are somewhat different, the underlying physical principles are equal [17] and thus it makes sense to refer to these methods using the most general term of QELS.

\section{Method Description}

2.1 Theoretical Basis of the Laser Speckle Methods
Among the dynamic processes in the body, one of the most prominent and best studied is blood circulation. QELS methods are suitable for the characterization of blood flow, where moving RBCs act as dynamic scatterers. In 1972 Riva et al. have demonstrated the applicability of the QELS for the measurements of blood flow in the retinal arteries, as well as whole blood flowing through capillary tubes [18]. Even in the presence of multiple scattering by RBCs, the authors were able to obtain a linear relation of the flow rate and frequency of the peak in the intensity fluctuations spectrum. Three years later Stern performed measurements of the microcirculation in the finger [19]. Due to the plurality of the flow directions and speeds of flow in the microvascular network no peak has been observed in the spectrum of the intensity fluctuations; however, energy contained in the low-frequency part can be used to characterize perfusion flow. Further on, Stern et al. reported observations of the pulsatile component in the Doppler system and studied the blood perfusion of the outer cortex of the rat kidney in response to the application of several drugs [20]. Later on Bonner and Nassal [21] have developed a model, which laid the theoretical ground for the application of LDF for the microcirculation analysis.

In the beginning of the 1980s, Fercher and Briers [22, 23] suggested to use the amount of speckle image blurring for the estimation of the correlation time of intensity fluctuations. The dynamics of the speckle pattern affects the obtained image if the decorrelation time is comparable to the exposure time $T$ of the camera since the speckle pattern evolves during the exposure. This leads to the blurring of the speckle and decreases the contrast of speckle image as detected by the camera. This approach has been coined Laser Speckle Contrast Analysis (LASCA) since it employs the spatial contrast (standard deviation of the time-integrated intensity pattern divided by the mean intensity) as a measure of the blurring:

$$
K(T)=\frac{\sigma\left(I_{T}\right)}{\left\langle I_{T}\right\rangle}=\sqrt{\frac{\left\langle I_{T}^{2}\right\rangle}{\left\langle I_{T}\right\rangle^{2}}-1}
$$


where $I_{T}$ denotes the intensity integrated over the time period $T$, $\sigma\left(I_{T}\right)$ is its standard deviation, and $\langle\ldots\rangle$ refers to means obtained over the spatial pixels.

With faster dynamics, and thus shorter correlation time of the intensity fluctuations, the effect of blurring becomes larger and the contrast $K$ decreases. This relation between contrast and correlation function can be quantified in the following way [24-26] (in order to keep the notation simple we will discuss the properties of the square of contrast $K^{2}$ ):

$$
K^{2}(T)=\frac{2}{T} \int_{0}^{T}\left[g_{2}(\tau)-1\right]\left(1-\frac{\tau}{T}\right) d \tau=\frac{2 \beta}{T} \int_{0}^{T}\left|g_{1}(\tau)\right|^{2}\left(1-\frac{\tau}{T}\right) d \tau
$$

For some specific simple forms of the correlation function analytic solutions can be derived for the correlation time dependence $K$ [25].

For example, for a single exponential decay of the correlation function $g_{1}^{s}(\tau)=\exp \left(-\tau / \tau_{\mathrm{c}}\right)$, where $\tau_{\mathrm{c}}$ is called correlation time, the contrast is given by the following expression [25]:

$$
K^{2}(T)=\beta[\exp (-2 x)-1+2 x] / 2 x^{2},
$$

where $x=T / \tau_{\mathrm{c}}$.

For the case of DWS backscattering from a turbid medium undergoing simple Brownian motion, the field correlation function is given by the following equation $\mathfrak{g}_{1}^{\mathrm{m}}(\tau)=\exp \left(-\gamma \sqrt{6 \tau / \tau_{0}}\right)$ and one finds for the contrast

$$
K^{2}(T)=\beta[(3+6 \sqrt{x}+4 x) \exp (-2 \sqrt{x})-3+2 x] / 2 x^{2}
$$

where $x=6 \gamma^{2} T / \tau_{0}$.

Both expressions essentially assume a single diffusive relaxation process such as provided by Brownian motion of monosized micron scale objects dispersed uniformly in a medium. Within that framework the former result of (6) is exact in the case of single scattering while the latter (7) is a very good approximation for the case of multiple scattering. One should keep in mind that the assumptions made are certainly much too simple given the complexity of real tissue structure and dynamics. Nevertheless, given the simplicity of the results and the lack of more sophisticated models, both equations have been used in the past to analyze LSI on experimental animals.

The experimental arrangement for the speckle contrast imaging is relatively simple. A quantitative interpretation of the contrast values requires some assumptions concerning the particular shape of the correlation function and the impact of a static component in the detected signal [27]. In addition, the contrast has the maximal sensitivity (with respect to the underlying relaxation processes) if the correlation time is of the order of the exposure time [28] and it is significantly reduced if the exposure time is much smaller or much larger than the correlation time. 
In this limit a conversion of the contrast to the correlation values can be performed only with significant error.

Since the original work by Riva et al. [18] hundreds of scientific papers have been published on various applications of light scattering methods to resolve physiological questions. A number of companies have successfully commercialized the QELS systems for monitoring the microcirculation, e.g., Moor Instruments Inc. (UK), Perimed Instruments AB (Sweden), Aimago SA (Switzerland). The majority of these devices are based on the laser Doppler paradigm; however, the LSI system has also been commercialized recently.

Nevertheless, the DLS methods are still suffering from several important drawbacks, which impede their penetration to the regular clinical practice. The major problem is the absence of a quantitative link with the real physiological parameters of microvascular flow, expressed in common units. Instead most of the devices provide relative units. Another drawback is the significant temporal and spatial variability of the measurements. The temporal variability is usually related to the intrinsic non-stationarity of the flow, which is changing during a heartbeat cycle, breath, and the dynamic processes in the tissue itself, which is also called biological zero. The spatial variability is usually related to the small sampling volume of the fiber system (within $1 \mathrm{~mm}^{3}$ ), which is affected by the local structure of the vasculature and the relative amount of Dopplershifted photons in the signal [29].

The so-called non-ergodicity problem might arise if the detected light consists of components with different dynamics, e.g., light scattered by moving RBCs and light only reflected from the static tissues or bone. The non-ergodicity of the signal is not addressed in the proper way if the impact of the non-shifted photons is ignored in the analysis, which is often the case. This in turn results in the incorrect estimate of blood flow.

A correlation-based analysis of QELS signals can potentially address some of the problems mentioned above since an extensive theory and various experimental techniques have been developed for the quantitative interpretation of data, also addressing the problem of non-ergodicity [30-32]. For example, it is essential to analyze the speckle statistics not only in temporal but also in spatial domain, which is the basis of the so-called multi-speckle technique [33-35]. The original LASCA method based on the spatial speckle statistics ensures ergodic averaging, since the speckle size on the detector is adjusted to the pixel size and calculation within LASCA window $N \times N$ performs averaging over up to $N^{2}$ statistically independent speckles. Unfortunately, simplifications and assumptions of LASCA are also sources of drawbacks, e.g., the original method is not able to properly analyze the speckle field with multiple dynamic components, for example, consisting of a dynamic contribution from moving RBCs, a slow evolving component caused by vasomotion, and the static contribution from the rigid tissues or bone. 


\subsection{Dynamic Laser Speckle Imaging}

However, it has been demonstrated that with the help of the additional temporal analysis [36] or using images obtained with different exposure times [37] the static component can be separated from the contrast signal. This is the main idea underpinning the approach of dynamic laser speckle imaging (dLSI), which is discussed in the next section.

There exist a number of practically important experimental situations, when the scattering fields of interest are mixed with the nonfluctuating electric fields scattered from static regions. A prominent example is the measurement with a strong static background or dynamical imaging of a volume covered by a static scattering surface. This is the case, for example, for a layered medium with dynamics localized in a deeper layer (e.g., when imaging through the skull).

Although the removal of the dura mater to access the brain cortex is still required in most cases, some groups perform imaging through the thinned skull of rats or through the intact skull of mice $[36,38-41]$. This has considerable advantages compared to the open skull experiments, as surgery is simplified and undesired side effects, such as cortical swelling, are avoided.

The influence of the skull on the laser speckle signal has been quantitatively addressed recently $[27,37]$. The presence of a static component in the speckle image affects the shape of the field correlation function by introducing a nonzero baseline which results in the overestimation of the contrast [27]. One of the efficient approaches to separate dynamic and static components is the multiexposure technique [37, 42-44]. Essentially, this technique performs the contrast analysis of the speckle fields integrated with different exposure times instead of a single one, used in the standard LASCA. The images obtained with longer exposure times provide information on the slow dynamics, while shorter times-on faster dynamics. The contrast of the image sampled with large exposure time gives an indication of the contrast baseline introduced by the static or pseudo-static components that can be subsequently subtracted from the contrast of images with shorter exposure in order to provide the quantitatively correct estimation of the blood flow dynamics. In order to obtain multi-exposed images the gating of the laser beam can be performed using, e.g., an acousto-optic modulator, while the camera is kept with the constant exposure [37]. This allows obtaining the images with constant intensity level while changing the exposure during the measurements.

We have recently proposed [36] a somewhat simpler processing scheme using a single exposure time which we called dLSI. dLSI allows accounting for static contribution to the optical signal and thus facilitates correct qualitative and quantitative interpretation of CBF. At the same time it does not require modifications of the typical technically simple LASCA experimental settings and image acquisition procedure, which is a major advantage of this approach. 
Contributions of the static and dynamic components to the contrast cannot be distinguished in a traditional LASCA approach since one obtains the weighted integral of the field correlation function $[22,23]$. It is however possible to derive a rather simple formalism to deal with the dynamics of intensity fluctuations in the presence of a static component. We can define the static contribution as the light that has been scattered by rigid structures only (such as bone or a skin layer which is not highly perfused with blood). The detected intensity in this case is composed of a nonfluctuating static part $I_{\mathrm{s}}$ and a dynamic part $I_{\mathrm{d}}$ with a relative static contribution to the total intensity: $\rho=\left\langle I_{\mathrm{s}}\right\rangle /\left(\left\langle I_{\mathrm{s}}\right\rangle+\left\langle I_{\mathrm{d}}\right\rangle\right)$. In this case the correlation function of the mixed field can be written as a composition of the correlation function of the pure dynamic part $g_{1 \mathrm{~d}}(\tau)$ and the time-independent baseline $\rho$ from static light $[27,45]$ :

$$
\mathscr{g}_{1}(\tau)=(1-\rho)\left|\mathscr{g}_{1 \mathrm{~d}}(\tau)\right|+\rho
$$

The spatial contrast of the superposition of static and dynamic speckles can be found by substituting (8) into (5):

$$
\begin{aligned}
K^{2}(T) & =\frac{2 \beta}{T} \int_{0}^{T}\left[(1-\rho)\left|\mathscr{g}_{1 \mathrm{~d}}(\tau)\right|+\rho\right]^{2}\left(1-\frac{\tau}{T}\right) \mathrm{d} \tau \\
& =\beta\left[(1-\rho)^{2} K_{2 \mathrm{~d}}^{2}(T)+2 \rho(1-\rho) K_{1 \mathrm{~d}}^{2}(T)+\rho^{2}\right]
\end{aligned}
$$

where the $K_{1 \mathrm{~d}}^{2}$ and $K_{2 \mathrm{~d}}^{2}$ represent the contrast of the dynamic field and dynamic intensity, respectively:

$$
\begin{aligned}
& K_{1 \mathrm{~d}}^{2}(T)=\frac{2}{T} \int_{0}^{T}\left|\mathscr{g}_{1 \mathrm{~d}}(\tau)\right|\left(1-\frac{\tau}{T}\right) \mathrm{d} \tau \\
& K_{2 \mathrm{~d}}^{2}(T)=\frac{2}{T} \int_{0}^{T}\left|g_{1 \mathrm{~d}}(\tau)\right|^{2}\left(1-\frac{\tau}{T}\right) \mathrm{d} \tau
\end{aligned}
$$

Together $K_{1 \mathrm{~d}}^{2}$ and $K_{2 \mathrm{~d}}^{2}$ define the mixed dynamic contrast:

$$
K_{12 \mathrm{~d}}^{2}(T)=\beta\left[(1-\rho)^{2} K_{2 \mathrm{~d}}^{2}(T)+2 \rho(1-\rho) K_{1 \mathrm{~d}}^{2}(T)\right]
$$

Since $K_{12 \mathrm{~d}}^{2}(T)$ is dependent on $\rho$ it is not a pure measure of dynamics. However, it decays to zero for the infinitely short correlation time and reaches the maximum of $\beta\left(1-\rho^{2}\right)$ in the limit of infinitely long correlation time. The total measured contrast can be also written as following:

$$
K^{2}(T)=K_{12 \mathrm{~d}}^{2}(T)+\beta \rho^{2}
$$

In the absence of a static component $(\rho=0)(9)$ reduces to (5) and the mixed dynamic contrast equals dynamic intensity contrast $K_{2 \mathrm{~d}}^{2}$.

In an LSI experiment the exposure time $T$ (typically from 1 to $20 \mathrm{~ms}$, [28]) is chosen to be larger than the intensity correlation time $\tau_{\mathrm{c}}$ (in the range $0.001-0.1 \mathrm{~ms}$ for $\mathrm{CBF}$ ). The time interval between successive frames $\Delta t$ is for technical reasons larger than $T$ and we find $\Delta t>T>>\tau_{\mathrm{c}}$. We can than assume that $\mathscr{g}_{\mathrm{ld}}(\Delta t) \approx 0$. 
Hence two speckle patterns detected in two sequential frames are correlated only due to the presence of static speckles. We can thus find the amount of static light in the detected signal by comparison of sequential frames and by making use of a multi-speckle averaging scheme $[27,46]$.

With the correlation of dynamic intensity close to zero in (8) at lag time $\Delta t$ we can experimentally obtain the static contribution from the correlation of speckle patterns recorded in sequential camera frames:

$$
\rho^{2}=\left[g_{2}(\Delta t)-1\right] / \beta
$$

Since the contribution of the static light can be positiondependent, $\rho$ must be computed locally as well.

One can introduce the obtained $\rho$ into (9) in order to relate the experimentally obtained contrast $K^{2}$ to the correlation time $\tau_{c}$ or relaxation time $\tau_{0}$ of the dynamic part of the correlation function defined by DLS or DWS models. This can be done numerically by calculating the function $K^{2}\left(\tau_{c}\right)$ or $K^{2}\left(\tau_{0}\right)$ and taking $\rho$ into account. Instead of integrating the correlation function in (9), one can use the analytical expressions for the dependence of dynamic contrasts $K_{1 d}{ }^{2}$ and $K_{2 \mathrm{~d}}{ }^{2}$ on corresponding relaxation times, available for the simplest functional forms of field correlation function (such as single or stretched exponential) [25]. Box 1 provides a step-by-step guide for implementation of the dLSI method.

\section{Box 1 dLSI Algorithm}

Further we present a step-by-step guide for implementation of the dLSI method to the laser speckle data.

Step 1: Mixed dynamic contrast

The local mixed dynamic contrast $K^{2}{ }_{12 \mathrm{~d}}$ can be found for the set of pixels $i=1 \cdots N$ as following:

$$
K_{12 \mathrm{~d}}^{2}=\frac{\left\langle\left(I_{2}-I_{1}\right)^{2}\right\rangle}{2\left\langle I_{1}\right\rangle\left\langle I_{2}\right\rangle}
$$

where $\langle\cdots\rangle$ denotes at least the spatial averaging over a selected set of pixels which can be extended with temporal statistics collected over the same pixels. Speckle intensities $I_{1}\left(x_{i}\right)$ and $I_{2}\left(x_{i}\right)$ with $i=1 \ldots N$ defining the same set of pixels represent two time separated speckle images (for example, sequential images). The mean intensities are found from averaging over the corresponding set of pixels:

$$
\langle I\rangle=\frac{1}{N} \sum_{i=1}^{N} I\left(x_{i}\right)
$$

and

$$
\left\langle\left(I_{2}-I_{1}\right)^{2}\right\rangle=\frac{1}{N} \sum_{i=1}^{N}\left[I_{2}\left(x_{i}\right)-I_{1}\left(x_{i}\right)\right]^{2}
$$

(continued) 


\section{Box 1 (continued)}

Equation (14) implies that the square of the mixed dynamic contrast is equal to half of the mean squared difference of the sequential image frames normalized with the product of the mean intensities of those frames or the so-called second-order structure function [47] at the inter-frame time. The mixed contrast itself provides a good measure of the overall dynamics in the presence of the static component, since it eliminates the pure static baseline in (9). It can be used to generate the maps of dynamics if the quantitative interpretation is less crucial.

\section{Step 2: Static contribution}

As can be seen from (11) the dynamic contrast $K_{12 \mathrm{~d}^{2}}$ still includes the unknown static contribution $\rho$, which is required for the further quantitative conversion to the correlation time. The static contribution can be found with an additional processing step using the same speckle patterns $I_{1}$ and $I_{2}$ similar to the step 1 :

$$
\rho=\frac{1}{\beta^{1 / 2}}\left[\frac{\left\langle I_{1} I_{2}\right\rangle}{\left\langle I_{1}\right\rangle\left\langle I_{2}\right\rangle}-1\right]^{1 / 2}
$$

where

$$
\left\langle I_{1} I_{2}\right\rangle=\frac{1}{N} \sum_{i=1}^{N} I_{1}\left(x_{i}\right) I_{2}\left(x_{i}\right)
$$

is the mean product of the intensities at the pixels on sequential images. Equation (15) is essentially the multi-speckle calculation of the correlation of images $I_{1}$ and $I_{2}$.

The coherence factor $\beta$ used in this calculation has to be obtained separately. It can be calibrated using a solid white medium such as a block of Teflon with $\rho=1$.

Step 3: Conversion to correlation times

Using the previously obtained static contribution $\rho$ and assuming a certain shape of the dynamic field correlation function $g_{1 d}(\tau)$ (e.g., single or stretched exponent), we can find theoretical dependence of dynamic contrast $K^{2}{ }_{12 \mathrm{~d}}$ on the corresponding correlation or relaxation time. Using the value of mixed dynamic contrast obtained on step 1 we can numerically invert this relation to obtain the correlation time.

These steps should be repeated for all local pixel subsets composing the images in order to obtain the maps of the correlation time.

It has been demonstrated [36] that with the dLSI approach it is possible to perform functional LSI through the thinned or intact skull of rats with higher accuracy.

\subsection{Experimental Setup for Laser Speckle Imaging}

2.3.1 Animal Preparation
Surgery is preferentially performed under isoflurane anesthesia, whereas during the actual experiment the rodents should be anesthetized with $\alpha$-chloralose (s.c.). Catheters are placed in a femoral artery and vein for the continuous monitoring of the arterial blood pressure (90$110 \mathrm{mmHg}$ ) and for the administration of pharmacological agents. In our lab, animals are normally tracheotomized and mechanically ventilated to maintain physiological arterial levels of $\mathrm{pH}(7.35-7.45), \mathrm{pCO}_{2}$ (34-38 $\mathrm{mmHg})$, and $\mathrm{pO}_{2}(>100 \mathrm{mmHg})$. The body temperature is held constant at $37{ }^{\circ} \mathrm{C}$ using a regulated heating pad. During the 


\subsubsection{Optical Imaging}

measurements, animals are placed in a stereotactic frame (David Kopf Instruments, Tujunga, USA). In our hands, more reproducible and stable results are obtained with the thinned bone preparation. For this, the skull covering the cortex is carefully thinned to translucency using a surgical drill (Osseodoc, Bien-Air Surgery S.A. Le Noirmont, Switzerland). The thinned bone is then covered with $1 \%$ agar and a round cover glass (approximately $5 \mathrm{~mm}$ diameter for the use in rats, smaller for mice). The cover slip prevents drying of the agar. To avoid reflections, it should be placed parallel to the imaging plane.

For single whisker stimulation, we insert one vibrissa into a cannula, which is attached to a piezoelectric ceramic wafer (Piezo Systems Inc., Cambridge, MA, USA). A computer-triggered pulse $(50 \mathrm{~ms})$ sent to the wafer produces a $1 \mathrm{~mm}$ rostral to caudal deflection of the vibrissa. The stimulation lasts $4 \mathrm{~s}$ with a frequency of $4 \mathrm{~Hz}$. Before stimulation a baseline phase of at least $2 \mathrm{~s}$ should be acquired. A $60 \mathrm{~s}$ inter-trial interval is recommended to allow the hemodynamic signal to return to baseline.

Cortical images can be acquired using a CCD camera (we use a 12-bit Pixelfly VGA camera, PCO Imaging, Kelheim, Germany). Either a custom-made tandem lens system or a stereomicroscope (e.g., Leica MZ16 FA, Leica Microsystems, Heerbrugg, Switzerland) can be used for imaging. We focus $0.5 \mathrm{~mm}$ below the cortical surface. Collimated $785 \mathrm{~nm}$ laser light is shown onto the imaged cortical area (e.g., TuiOptics, Munich, Germany). We normally use free beam optics (mirrors and lenses) to collimate and steer the light (see Fig. 2). The images $(640 \times 480$ pixels $)$ are

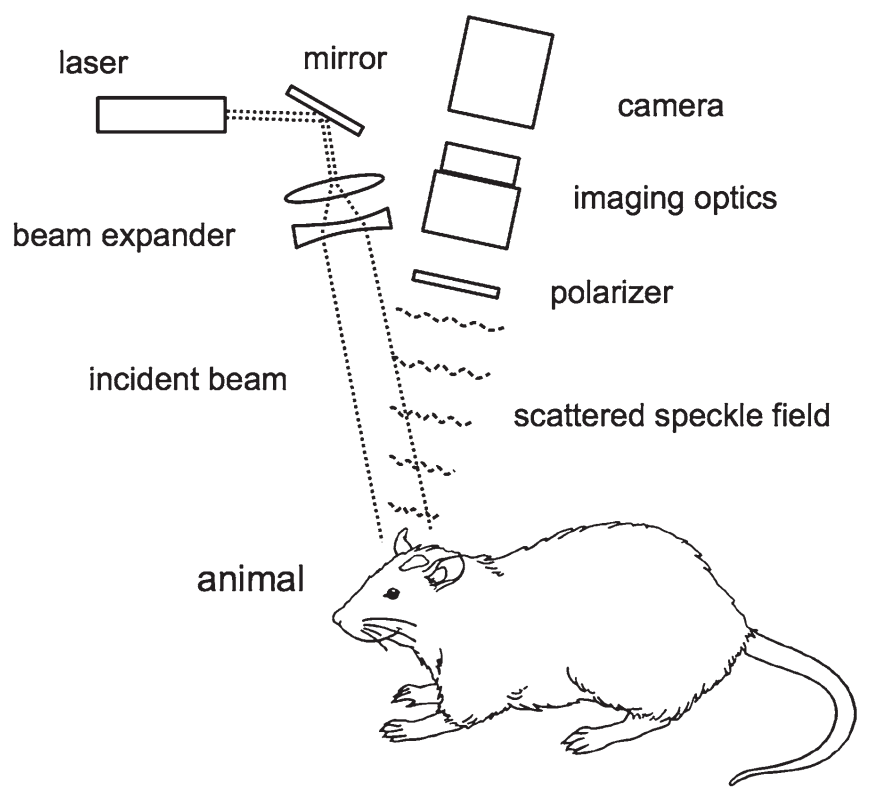

Fig. 2 Typical experimental setup for the laser speckle imaging. Coherent light from the laser is expanded and used to illuminate the tissue. Diffusively scattered light forms a speckle field, which produces an image on the camera through the polarizer and imaging optics. 


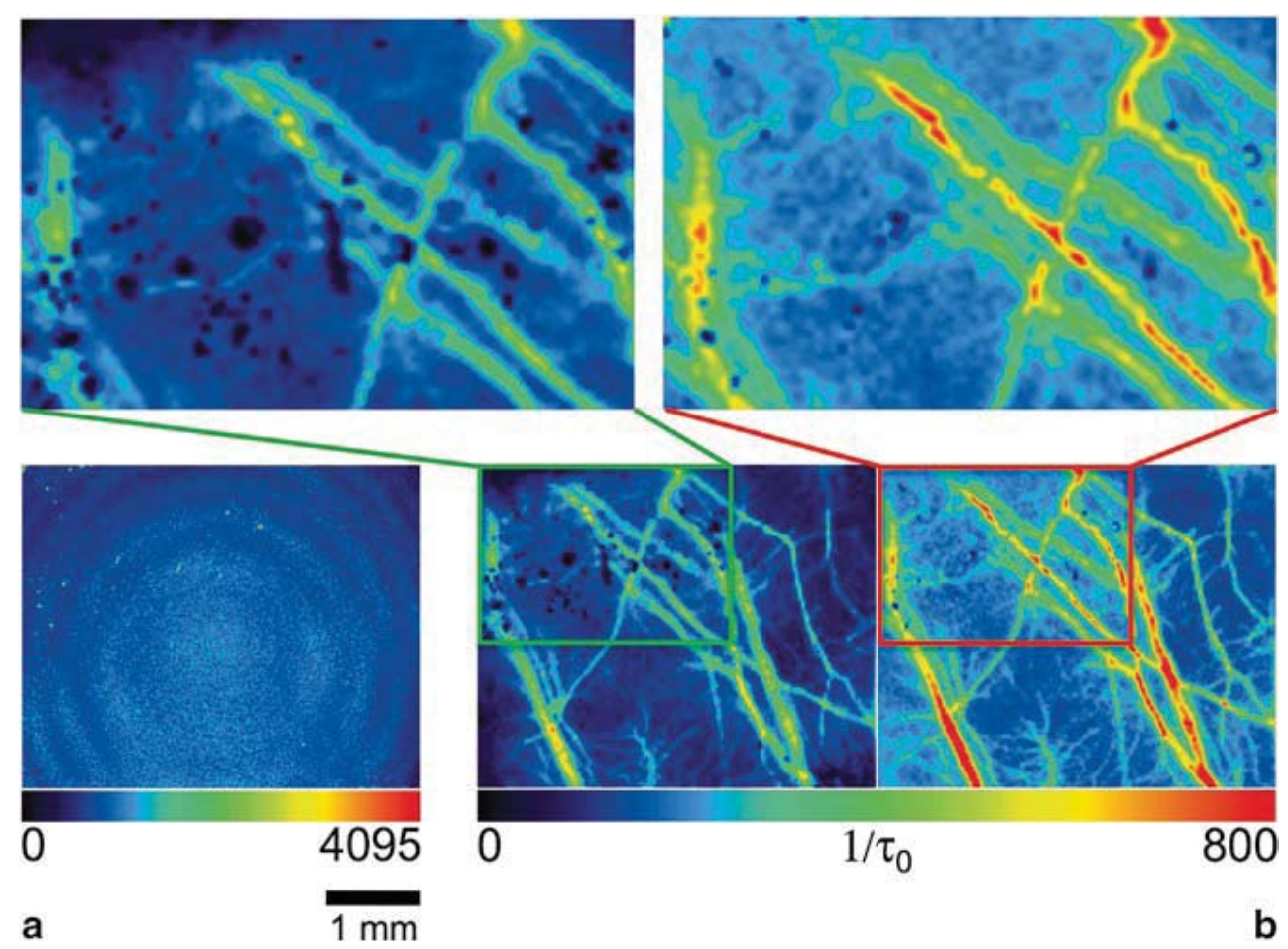

Fig. 3 (a) Raw speckle reflection recorded with $10 \mathrm{~ms}$ exposure time, measured through thinned skull. (b) Left. Reciprocal correlation time (RCT) derived from classical LASCA. (b) Right. RCT computed with dynamic laser speckle imaging. Note the significant reduction of specular reflections and effects of uneven illumination in the right panel. From [36].

\subsection{Laser Speckle Imaging in the Rat Somatosensory Cortex}

acquired with a frame rate of around $50 \mathrm{~Hz}$. For most of the applications, our camera specifications are sufficient; however, a larger number of pixels as well as a higher frame rate could be advantageous in certain situations. An exposure time of $10 \mathrm{~ms}$ is ideal, although other groups proposed slightly different values [28]. The f-stop of the imaging optics should be adjusted to find a best compromise between speckle contrast and number of speckles [48]. This is accomplished when the size of the speckles is approximately identical to the pixel size of the image sensor [10].

The raw reflection signal from the somatosensory cortex is shown in Fig. 3a. Applying either the classical LASCA or dLSI algorithm yields the flow map depicted in Fig. 3b, where the surface vessels are easy to identify. The relative signal change following the 4-s stimulation is shown in Fig. 4, where a circumscribed region shows a marked increase in CBF reflected by the increase in change in the reciprocal correlation time $\left(\Delta \tau_{0}^{-1}\right)$, estimated from DWS approximation of $(7)$. The time-activity curve shows an increase in the hemodynamic signal with a delay of approximately $0.5 \mathrm{~s}$. The peak amplitude is reached shortly before the end of the stimulation period. It is also noteworthy that the area of increased blood flow extends over a region that is considerably larger than the known barrel size, which is in the order of $0.4 \mathrm{~mm}$ for the principal whisker $\mathrm{Cl}$. 


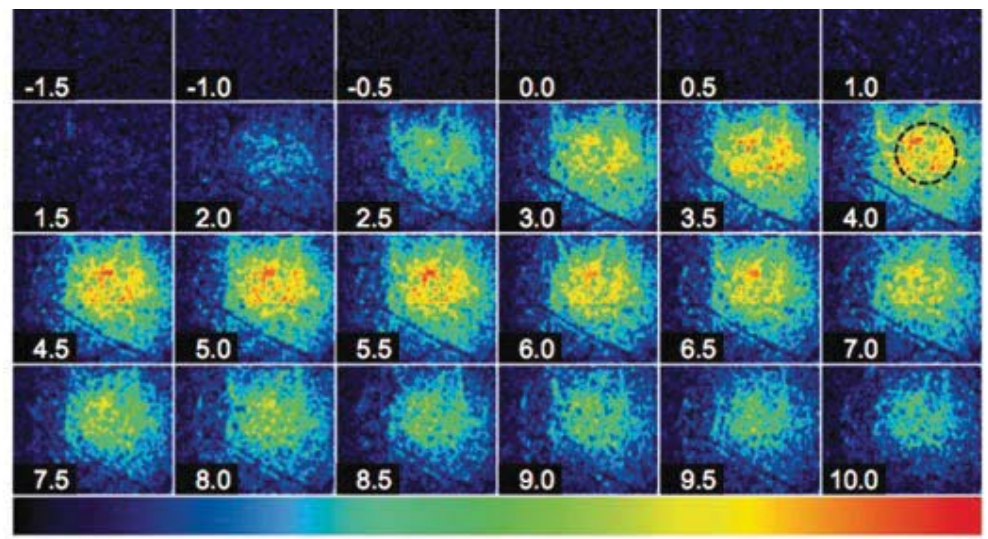

b

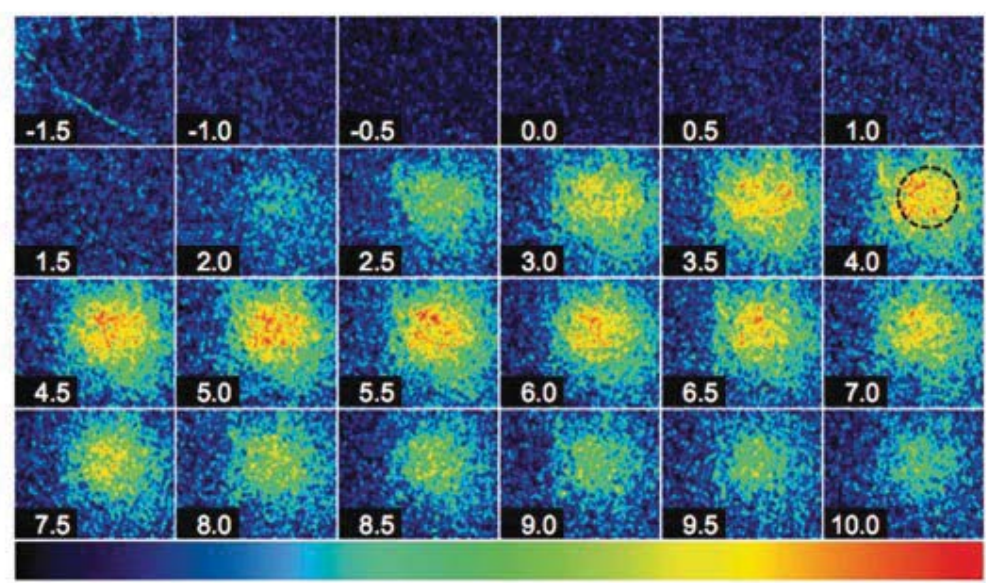

0

$\Delta 1 / \tau_{0}[\%]$

25

$4 \mathrm{~mm}$

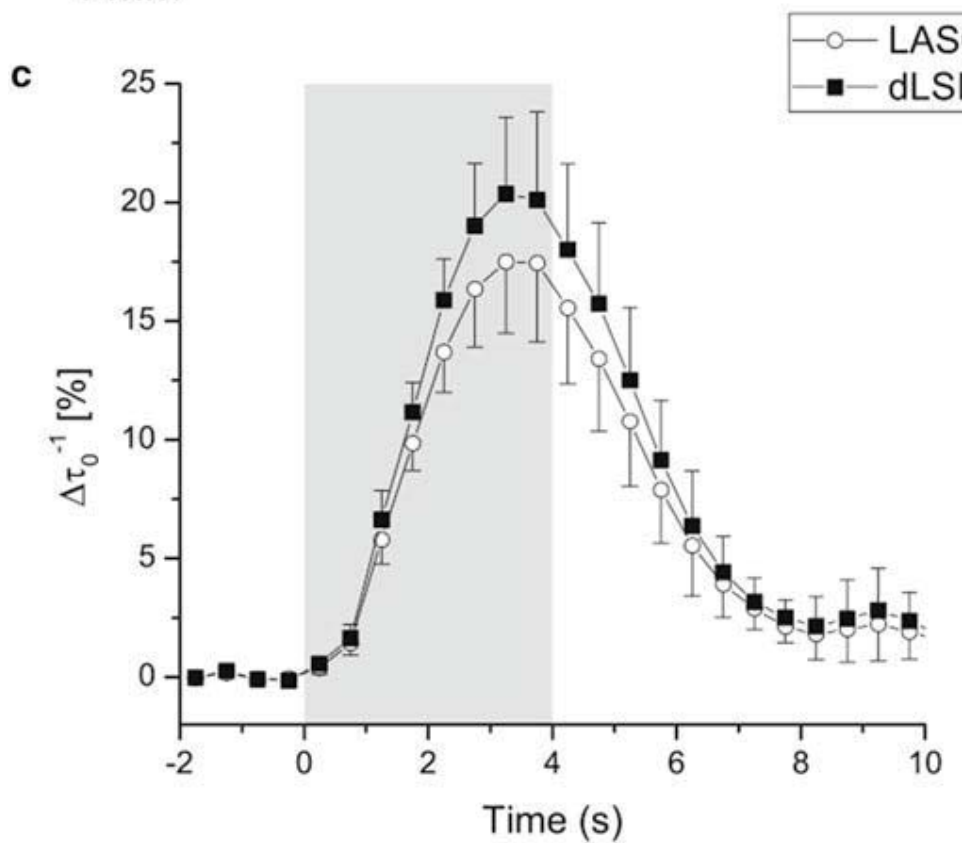

Fig. 4 Dynamic laser speckle imaging in the somatosensory cortex during single vibrissa (C1) stimulation. Maps represent relative changes of the RCT obtained from LASCA (a) and dLSI (b) signal upon single whisker stimulation (time step $0.5 \mathrm{~s}$ ). (c) Time-activity curves averaged over 5 animals (10 trials each) from activated area (lines show average; error bars represent standard error of the mean). Gray area represents the duration of the whisker stimulation. Modified from [36]. 